\title{
Discrete element model for crack propagation in brittle materials
}

\author{
Ba Danh Le, Georg Koval ${ }^{* \dagger}$ and Cyrille Chazallon \\ ICUBE Laboratory, UMR 7357, CNRS, National Institute of Applied Sciences of Strasbourg, 24 Boulevard de la \\ Victoire, 67084, Strasbourg, France
}

\begin{abstract}
SUMMARY
We propose a discrete element model for brittle rupture. The material consists of a bidimensional set of closed-packed particles in contact. We explore the isotropic elastic behavior of this regular structure to derive a rupture criterion compatible to continuum mechanics. We introduce a classical criterion of mixed mode crack propagation based on the value of the stress intensity factors, obtained by the analysis of two adjacent contacts near a crack tip. Hence, the toughness becomes a direct parameter of the model, without any calibration procedure. We verify the consistency of the formulation as well as its convergence by comparison with theoretical solutions of tensile cracks, a pre-cracked beam, and an inclined crack under biaxial stress. Copyright () 2015 John Wiley \& Sons, Ltd.
\end{abstract}

Received 4 March 2015; Revised 16 June 2015; Accepted 24 June 2015

KEY WORDS: brittle materials; discrete element method; crack propagation; fracture mechanics

\section{INTRODUCTION}

Real materials may present complex structure and uncountable flaws (cracks, property discontinuities, voids, etc.) which define their mechanical behavior. Theoretical approaches simplify the material description based on model parameters. In continuum mechanics, isotropic linear elasticity is described by only two parameters (e.g., Poisson's ratio $v$ and Young's modulus $E$ ) relating stresses to strains. If the elastic behavior is restrained to a certain range of stresses and strains, and no stress is conceivable outside this range, a quasi-brittle failure can be therefore associated. Under low stress (and strain) gradients, the rupture behavior depends basically on the strength of the material, which may be limited by some stress condition like in Rankine, Mohr-Coulomb, and Von Mises materials. On the other hand, under very high-stress gradients (e.g., induced by existing cracks), the rupture behavior is well described by fracture mechanics formulation. Crack propagation is mostly based on the evaluation of the energy release rate, which may be limited by the fracture energy of the material.

Various numerical methods from continuum mechanics have been adapted to study the fracture behavior of materials like the Barenblatt-Dugdale cohesive zone crack model [1,2] in the early 1960s, special finite elements (finite element method) proposed by Henshell and Shaw [3] in the 1970s, and more recently the work of Belytschko with the extended finite element method [4-6]. These widely used methods present very good results, while the number of cracks is relatively limited. For very complex systems of cracks, presenting fragmentation patterns (e.g., induced by very large strains or cyclic loading), discrete approaches become more competitive options.

The discrete element method (DEM) [7] was originally developed for modelling granular and particulate systems [8-13]. Further developments have adapted the method to study the fracture of quasi-brittle materials such as concrete and rocks [14-18]. DEM is not based on continuum

\footnotetext{
*Correspondence to: Georg Koval, Department of Civil Engineering and Topography, INSA of Strasbourg, 24 Boulevard de la Victoire, 67084, Strasbourg, France.

†E-mail: Georg.koval@insa-strasbourg.fr 
(a)

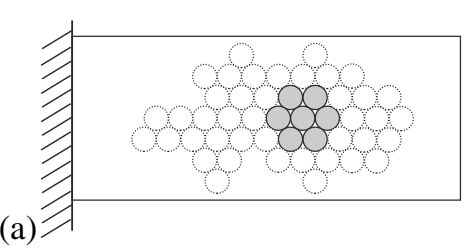

(b)

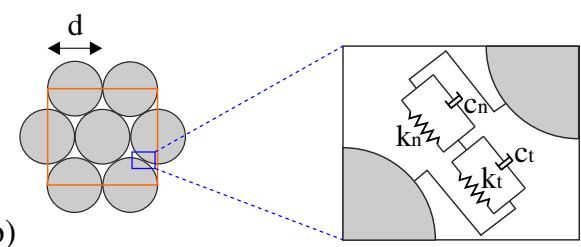

Figure 1. (a) Periodic compact distribution of particles to a discrete model and (b) the associated unit cell.

mechanics; materials are assimilated to assemblies of particles in contact (Figure 1). The constitutive parameters of these contacts (e.g., stiffness, strength, etc.) influence the behavior of the whole model at the macroscopic scale. These parameters are usually identified (calibrated) using some kind of optimization [19-21] intending to describe the expected behavior of the material (obtained experimentally, for example). This procedure allows the analysis of a specific range of experiments (or practical situations) previously considered during calibration (e.g., a sample under a bending moment should be calibrated considering tensile and compression tests). Despite the good concordance of discrete models with experimental results, it remains difficult to define the expected error of a prediction when no reference experimental data are available for the comparison. It is usually the case, because discrete element models are normally not conceived to present a convergent behavior associated with a systematic evolution of the discretization (as in continuum models), but an approximative behavior for a given sample configuration.

We aim to associate the advantages of continuous approaches like theoretical background and convergence toward a unique solution to a discrete approach. Recent works [22, 23] present analytical expressions which relate directly DEM contact parameters to elastic continuous solid parameters (i.e., Young's modulus and Poisson's ratio). Based on the existence of a relation between discrete and continuous models in elasticity, we propose a DEM approach for brittle materials. The concordance with classical theories (continuum mechanics) exempts us of any previous calibration of the model parameters in order to obtain convergent results.

This paper begins with a presentation of the contact law used in our discrete model and its relation with the material elasticity (Section 2). In Section 3, we present the theoretical elements of our discrete approach based on fracture mechanics. We compare our numerical results to classical cases of tensile fracture (mode I), shear fracture (mode II), and mixed mode in Section 4. Finally, we present the conclusions of our work.

\section{STRESS AND STRAIN ANALOGY IN ISOTROPIC ELASTICITY}

While cracks are not propagating, we can suppose that brittle materials present an elastic behavior. For a given problem, comparable results between continuous and discrete approaches must present similar stress and strain distributions. Let us consider a homogeneous (elastic and isotropic) material as an assembly of particles in contact, following the dense pack pattern shown in Figure 1(a). All mechanical properties of these particles are defined at the contact level (a local scale). The forces are transmitted through contacts, while material strain depends on particles translational and rotational motions.

\subsection{Contact model}

We adopt a linear relation between contact forces and relative particle position which can be seen as a simplified version of Hertz contact model (Figure 1). Let us define $\underline{\mathbf{n}}_{i j}$ as the normal vector pointing from the center of $i$ to the center of $j$ and $\underline{\mathbf{t}}_{i j}$ as the tangential vector, orthogonal to $\underline{\mathbf{n}}_{i j}$ and positively oriented shown in Figure 2.

The contact force (applied by a particle $j$ over a particle $i$ ) is decomposed in normal and tangential components (Figure 2), respectively $\underline{\mathbf{N}}_{i j}=\underline{\mathbf{N}}_{i j}^{e}+\underline{\mathbf{N}}_{i j}^{v}$ and $\underline{\mathbf{T}}_{i j}=\underline{\mathbf{T}}_{i j}^{e}+\underline{\mathbf{T}}_{i j}^{v}$, both depending on two contributions. 

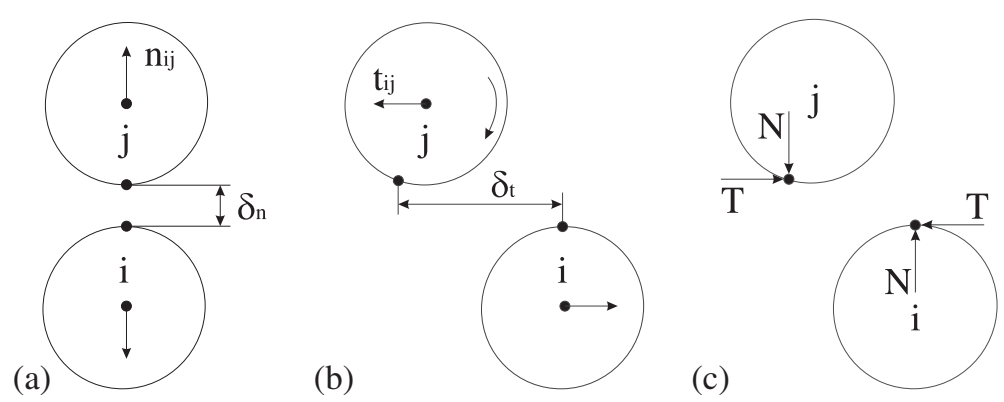

Figure 2. Normal displacement (a), tangential displacement, (b) and contact forces associated (c) between two particles $i$ and $j$.

The normal component is the sum of two contributions $\underline{\mathbf{N}}_{i j}=\underline{\mathbf{N}}_{i j}^{e}+\underline{\mathbf{N}}_{i j}^{v}$. The elastic one, defined as $\underline{\mathbf{N}}_{i j}^{e}=k_{n} \delta_{n} \underline{\mathbf{n}}_{i j}$, depends on the normal displacement $\delta_{n}$ and the normal stiffness $k_{n}$. The inelastic one, defined as $\underline{\mathbf{N}}_{i j}^{v}=c_{n} \dot{\delta}_{n} \underline{\mathbf{n}}_{i j}$ depends on a viscous damping parameter $c_{n}$ and the time derivative of the normal deflection $\dot{\delta}_{n}$.

Similarly, the elastic term of the tangential force, $\underline{\mathbf{T}}_{i j}^{e}=k_{t} \delta_{t} \underline{\mathbf{t}}_{i j}$ depends only on tangential relative displacement $\delta_{t}$ and tangential stiffness $k_{t}$. The inelastic term of the tangential force, $\underline{\mathbf{T}}_{i j}^{v}=c_{t} \dot{\delta}_{t} \mathbf{t}_{i j}$ is based on a tangential viscous damping parameter $c_{t}$ and on the time derivative of the tangential displacement $\dot{\delta}_{t}$. The latter contribution is only introduced to stabilize the numerical integration scheme. We choose $c_{n}, c_{t}$ as a small fraction of $\sqrt{m k_{n}}$ (where $m$ is the particle mass), which guarantees a negligible inelastic effect.

\subsection{Particle displacement and elastic behavior}

Our numerical approach is based on DEM like in [7, 13] alternating the computation of all contact forces and the application of Newton's second law to the particles. The discrete particle motion equations are solved by Gear's order three predictor-corrector algorithm [24]. We adopt a 2D close-packing distribution of particles, as shown in Figure 3(a), to represent a linear elastic isotropic material (Figure 3(b)). With a discrete approach, the relation between relative position of the particles and contact forces is analogous to strain and stress relation in a continuous approach. The balance of the contact forces gives rise to a macroscopic stress tensor. Equivalence between continuous and discrete approaches $[22,25]$ has shown that normal and tangential stiffness $\left(k_{n}\right.$ and $k_{t}$, respectively) can be directly related to elasticity parameters of the continuous solid (Young's modulus $E$ and Poisson's ration $v$, for example) as follows (in-plane stress):

$$
\left\{\begin{array}{l}
k_{n}=\frac{E}{\sqrt{3}(1-v)}, \\
k_{t}=\frac{1-3 v}{1+v} k_{n}=\frac{1-3 v}{\sqrt{3}\left(1-v^{2}\right)} E .
\end{array}\right.
$$

The relations expressed in Equation (1) were deduced for homogeneous stress and strain conditions. In more general conditions, where stress and strain gradients may be observed, they indicate asymptotic limits. Better correlations between continuous and discrete approaches are naturally obtained for materials described by smaller particles, which represent a more precise discretization.

(a)

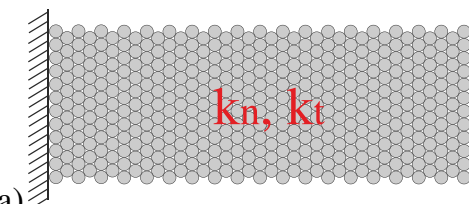

(b)

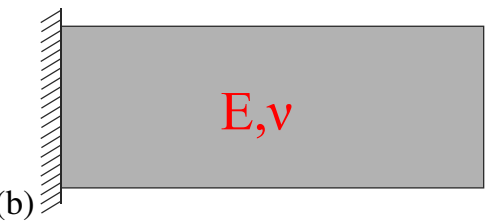

Figure 3. (a) Periodic structure of the discrete material and (b) continuous equivalence. 


\section{STRESS INTENSITY FACTORS AND CRACK PROPAGATION ANALOGY FOR BRITTLE MATERIALS}

The convergence similarity between discrete and continuous approaches in elasticity allows us to analyze the singular stress field at the vicinity of a crack (Figure 4) in order to extend the notion of stress intensity factors [26] and crack propagation to the discrete approach.

\subsection{Stress field near a crack tip and crack propagation}

Based on the polar coordinate system placed at the crack tip (Figure 4), the stress components can be written for $r>0$ as [27] follows:

$$
\left\{\begin{array}{l}
\sigma_{r r}(r, \theta)=\frac{K_{r r}(\theta)}{\sqrt{2 \pi r}}, \text { with } K_{r r}(\theta)=K_{I} \cos \frac{\theta}{2}\left(1+\sin ^{2} \frac{\theta}{2}\right)+K_{I I}\left(-\frac{5}{4} \sin \frac{\theta}{2}+\frac{3}{4} \sin \frac{3 \theta}{2}\right) \\
\sigma_{\theta \theta}(r, \theta)=\frac{K_{\theta \theta}(\theta)}{\sqrt{2 \pi r}}, \text { with } K_{\theta \theta}(\theta)=K_{I} \cos \frac{\theta}{2}\left(1-\sin ^{2} \frac{\theta}{2}\right)+K_{I I}\left(-\frac{3}{4} \sin \frac{\theta}{2}-\frac{3}{4} \sin \frac{3 \theta}{2}\right) \\
\sigma_{r \theta}(r, \theta)=\frac{K_{r \theta}(\theta)}{\sqrt{2 \pi r}}, \text { with } K_{r \theta}(\theta)=K_{I} \sin \frac{\theta}{2} \cos ^{2} \frac{\theta}{2}+K_{I I}\left(\frac{1}{4} \cos \frac{\theta}{2}+\frac{3}{4} \cos \frac{3 \theta}{2}\right)
\end{array}\right.
$$

The parameters $K_{I}$ and $K_{I I}$ are the so-called stress intensity factors. $K_{I}$ is associated with crack opening mode, while $K_{I I}$ is associated with shear mode. Their values, which depend on loading and crack shape, quantify the strength of the singularity. For brittle materials (in the domain of linear elastic fracture mechanics), they are directly associated with the energy release rate during crack extension [26]. Some crack propagation criteria for brittle materials take advantage of this principle, relating the stress intensity factors to the material toughness $K_{I C} . K_{r r}, K_{r \theta}$, and $K_{\theta \theta}$ are the effective stress intensity factors, and $\theta$ is the relative angle defined by the tangent direction at the crack tip. A free surface condition imposes in reality $\sigma_{r r}(0, \theta)=0$ and $\sigma_{r \theta}(0, \theta)=0$; consequently, $\sigma_{\theta \theta}(0, \theta)$ is a principal stress.

One of the simplest criteria of crack propagation in mixed mode was proposed by Erdogan et al. [28], which is based on the maximum circumferential tensile stress. This theory states that crack propagates perpendicularly to the direction of the greatest tension, following $\theta_{0}$. Considering the terms of Equation (2), that is to say $K_{\theta \theta}\left(\theta_{0}\right)=\max K_{\theta \theta}(\theta)$, and consequently $K_{r \theta}\left(\theta_{0}\right)=0$. For a given stress distribution, the stability of the crack depends therefore on the structure material. As long as $K_{\theta \theta}\left(\theta_{0}\right)<K_{I C}$, the crack remains stable, otherwise it propagates [27].

In the discrete medium, the efforts are transmitted by the contact forces between the particles. In the next section, we present the equivalence between the contact force (discrete medium) and the principal stresses (continuous medium). From the values of the principal stresses acting near the crack, we complete the construction of the model with a definition of the stress intensity factors for the discrete medium.

\subsection{Discrete crack analysis}

3.2.1. Mean stresses and strains in the discrete medium. We determine mean values of the components of the stress and strain tensor based on the behavior of one pair of contacts associated with three particles $(i, j$, and $k$ ) in contact: $k i$ and $k j$ (Figure 5(a)), near a crack under a given loading

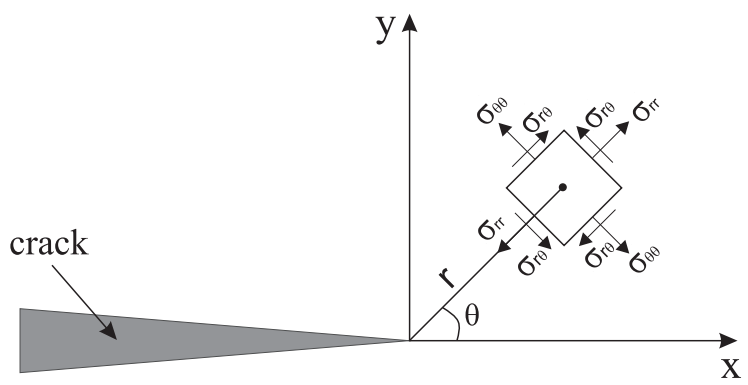

Figure 4. Crack tip and stresses near a crack tip. 


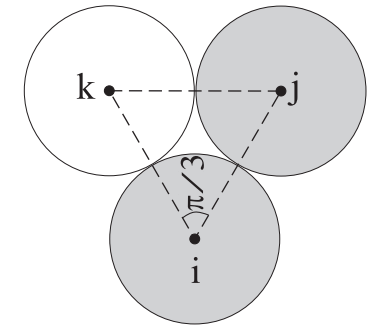

(a)

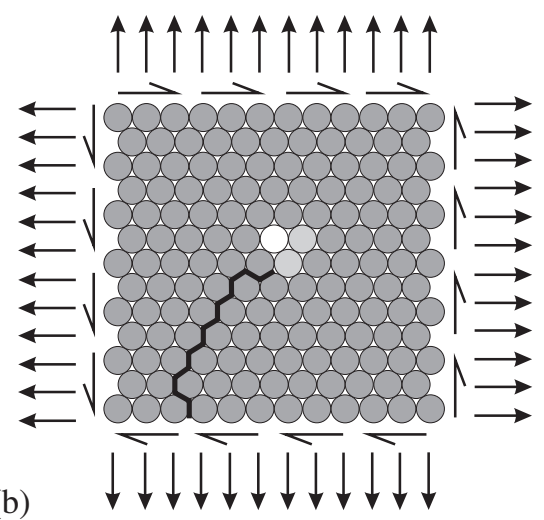

Figure 5. (a) The adjacent contacts at the crack tip in a (b) discrete medium.

(a)

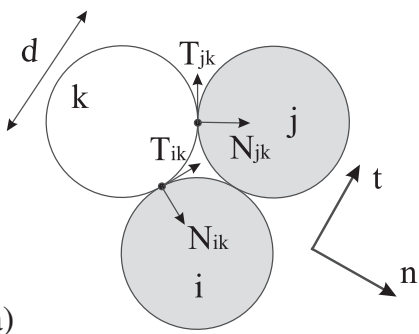

(b)

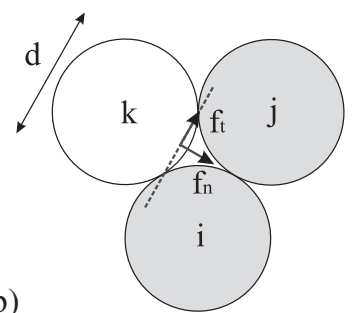

Figure 6. (a) Contact forces and (b) their resultants for an adjacent pair of contacts.

(Figure 5(b)). We define a local coordinate system $(n ; t)$, where $t$ virtually connects both contacts for which $n$ is an orthogonal axis.

The distribution of contact forces on the pair of contacts is shown in Figure 6(a); it has normal components $(N)$ and tangential components $(T)$. The components of the resultant contact force in $(n ; t)$ coordinate system $\left(f_{n}\right.$ and $f_{t}$, respectively; Figure $\left.6(\mathrm{~b})\right)$ can be written as follows:

$$
\left\{\begin{array}{l}
f_{n}=\frac{1}{2}\left(N_{i k} \sqrt{3}+T_{i k}+N_{j k} \sqrt{3}-T_{j k}\right), \\
f_{t}=\frac{1}{2}\left(-N_{i k}+T_{i k} \sqrt{3}+N_{j k}+T_{j k} \sqrt{3}\right) .
\end{array}\right.
$$

We can associate mean stresses to these forces, considering the length of a particle diameter $d$ :

$$
\left\{\begin{array}{l}
\bar{\sigma}_{n}=f_{n} / d \\
\bar{\sigma}_{t}=f_{t} / d
\end{array}\right.
$$

The contacts $k i$ and $k j$ may present normal and tangential displacements $\left(\delta_{n i k}\right.$ and $\delta_{t i k} ; \delta_{n j k}$ and $\delta_{t j k}$, respectively). Mean strain values $\bar{\varepsilon}_{n n}$ and $\bar{\varepsilon}_{t t}$ (Figure 7(a)) can be derived from these contact displacements, considering the dimensions of the particle structure (Figure 7(b)):

$$
\left\{\begin{array}{l}
\bar{\varepsilon}_{n n}=\frac{1}{2}\left(\delta_{n i k} \sqrt{3}+\delta_{n j k} \sqrt{3}+\delta_{t i k}-\delta_{t j k}\right) \frac{2}{d \sqrt{3}}, \\
\bar{\varepsilon}_{t t}=\frac{1}{2}\left(\delta_{n i k}+\delta_{n j k}-\delta_{t i k} \sqrt{3}+\delta_{t j k} \sqrt{3}\right) \frac{2}{d},
\end{array}\right.
$$

3.2.2. Principal stresses and strains. The complete stress tensor (in 2D) can be expressed by the principal stresses $\bar{\sigma}_{I}$ and $\bar{\sigma}_{I I}$ and their orientation. Let us call $\beta$ the angle between $(n ; t)$ and the coordinate system associated with the principal stresses (Figure 8(a)). This principle can be extended 
(a)

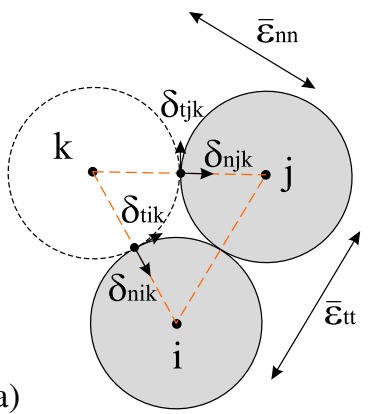

(b)

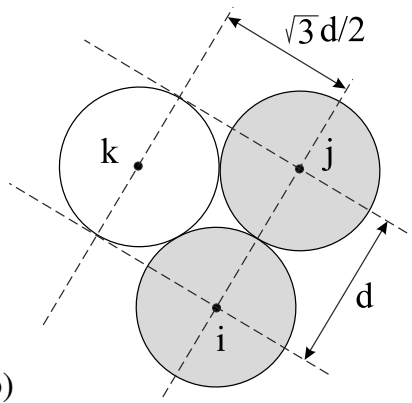

Figure 7. (a) Contact displacements, mean strains, and (b) associated dimensions of the particle structure.
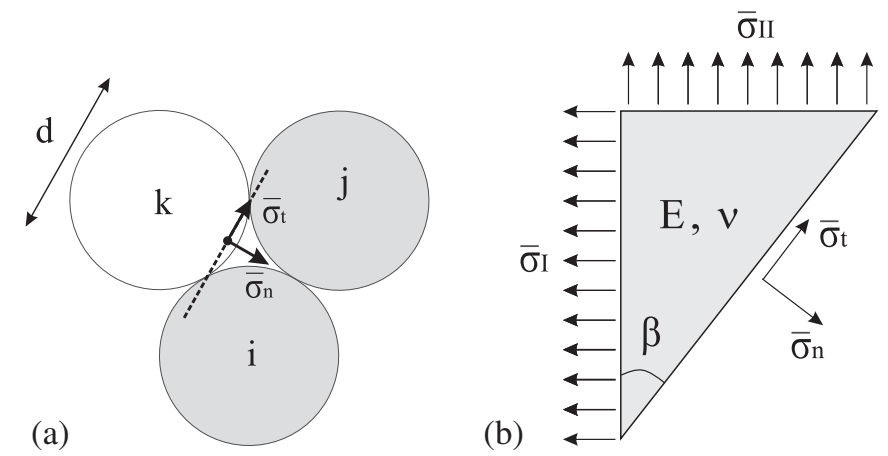

Figure 8. (a) Mean stresses $\bar{\sigma}_{n}$ and $\bar{\sigma}_{t}$, and (b) their relation to the stress field of an equivalent continuous medium.

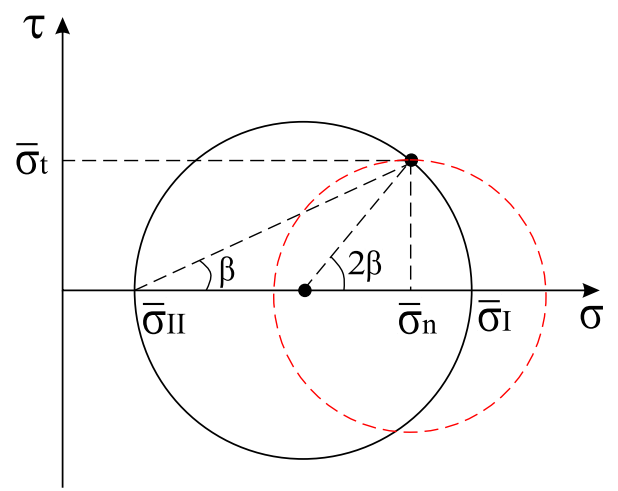

Figure 9. Mohr's circle associated on stress state $\bar{\sigma}_{n}$ and $\bar{\sigma}_{t}$.

to the mean stress values on the discrete medium (Figure 8(b)). After a force balance, the principal stresses may be calculated as

$$
\left\{\begin{array}{l}
\bar{\sigma}_{I}=\bar{\sigma}_{n}+\bar{\sigma}_{t} \tan (\beta), \\
\bar{\sigma}_{I I}=\bar{\sigma}_{n}-\bar{\sigma}_{t} / \tan (\beta) .
\end{array}\right.
$$

In the Mohr plane (Figure 9), we observe the infinite number of circles associated with a pair of stress values $\bar{\sigma}_{n}$ and $\bar{\sigma}_{t}$. For this reason, it is imperative to know the angle $\beta$ for the determination of mean principal stresses $\bar{\sigma}_{I}$ and $\bar{\sigma}_{I I}$. 
For a homogeneous material, the elastic behavior can be written as a function of Young's modulus $E$ and Poisson's ratio $v$

$$
\underline{\underline{\varepsilon}}=\frac{1}{E}[(1+v) \underline{\underline{\sigma}}-v \operatorname{tr}(\underline{\underline{\sigma}}) \underline{\underline{I}}]
$$

where $\underline{\underline{\varepsilon}}=\left[\begin{array}{cc}\bar{\varepsilon}_{I} & 0 \\ 0 & \bar{\varepsilon}_{I I}\end{array}\right]$ is the mean stresses tensor (in 2D); $\underline{\underline{\sigma}}=\left[\begin{array}{cc}\bar{\sigma}_{I} & 0 \\ 0 & \bar{\sigma}_{I I}\end{array}\right]$ is the mean strains tensor (in 2D); $\bar{\varepsilon}_{I}$ and $\bar{\varepsilon}_{I I}$ are mean principal strains; $\bar{\sigma}_{I}$ and $\bar{\sigma}_{I I}$ are mean principal stresses; and $\underline{\underline{I}}$ is identity matrix. The principal stresses are directly related to principal strains by the expression:

$$
\left[\begin{array}{cc}
\bar{\varepsilon}_{I} & 0 \\
0 & \bar{\varepsilon}_{I I}
\end{array}\right]=\frac{1+v}{E}\left[\begin{array}{cc}
\bar{\sigma}_{I} & 0 \\
0 & \bar{\sigma}_{I I}
\end{array}\right]-\frac{v}{E}\left(\bar{\sigma}_{I}+\bar{\sigma}_{I I}\right)\left[\begin{array}{ll}
1 & 0 \\
0 & 1
\end{array}\right]
$$

Any shear deformation is observed in the direction associated with the principal stresses. Therefore, $\beta$ also defines the direction of the mean principal strains (Figure 10).

The unit vectors that define the coordinate $(n, t)$ with principal coordinate (Figure 6(a)) can be written as a function of $\beta$ as: $\underline{n}=(\cos \beta,-\sin \beta)$ and $\underline{t}=(\sin \beta, \cos \beta)$. The mean strains $\bar{\varepsilon}_{n n}$ and $\bar{\varepsilon}_{t t}$ are related to the mean principal strains by the relations $\bar{\varepsilon}_{n n}=\underline{n} \cdot \underline{\underline{\varepsilon}} \cdot \underline{n}$ and $\bar{\varepsilon}_{t t}=\underline{t} \cdot \underline{\underline{\varepsilon}} \cdot \underline{t}$, we have

$$
\left\{\begin{array}{l}
\bar{\varepsilon}_{n n}=\bar{\varepsilon}_{I} \cos ^{2} \beta+\bar{\varepsilon}_{I I} \sin ^{2} \beta, \\
\bar{\varepsilon}_{t t}=\bar{\varepsilon}_{I} \sin ^{2} \beta+\bar{\varepsilon}_{I I} \cos ^{2} \beta .
\end{array}\right.
$$

Like the principal stresses, the determination of $\beta$ is necessary to define the mean principal strains $\bar{\varepsilon}_{I}$ and $\bar{\varepsilon}_{I I}$ from a pair of strains values $\bar{\varepsilon}_{n n}$ and $\bar{\varepsilon}_{t t}$ acting on a pair of contacts.

3.2.3. Determination of $\beta$. We can determine the value of $\beta$ by associating the information from mean strains and stresses at a pair of contacts. From the equations system 8 and 9 , we can write the trace of the strain tensor from the trace of the stress tensor

$$
\bar{\varepsilon}_{n n}+\bar{\varepsilon}_{t t}=\frac{1-v}{E}\left(\bar{\sigma}_{I}+\bar{\sigma}_{I I}\right)
$$

From the expressions of the mean principal stresses Equation (6), Equation (10) can be written simply as follows:

$$
\bar{\varepsilon}_{n n}+\bar{\varepsilon}_{t t}=\frac{1-v}{E}\left(\bar{\sigma}_{n}+\bar{\sigma}_{t} \tan \beta+\bar{\sigma}_{n}-\bar{\sigma}_{t} / \tan \beta\right) .
$$
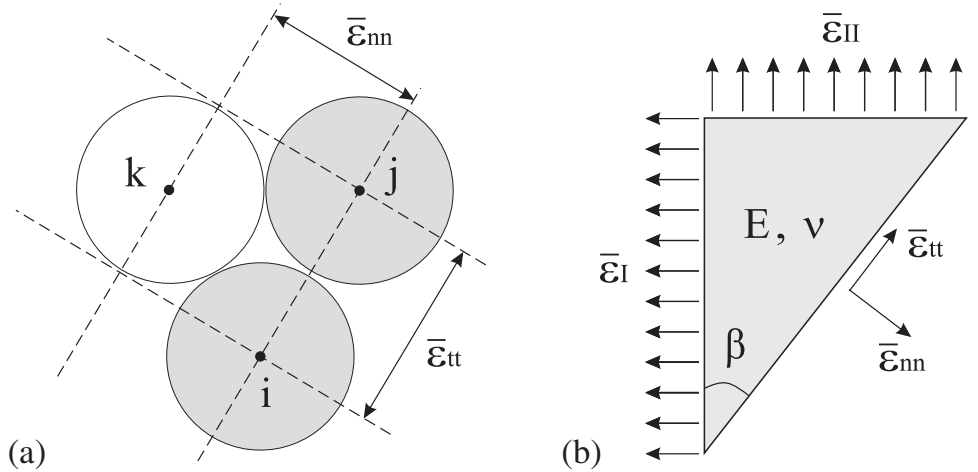

Figure 10. (a) Mean strain in a discrete medium and (b) in a continuous medium. 
We can simplify Equation (11) by isolating

$$
\tan \beta-\frac{1}{\tan \beta}=-\frac{2}{\tan (2 \beta)}=\frac{A}{\bar{\sigma}_{t}},
$$

where $A=\frac{E}{1-v}\left(\bar{\varepsilon}_{n n}+\bar{\varepsilon}_{t t}\right)-2 \bar{\sigma}_{n}$. Finally, the value of $\beta$ can be obtained

$$
\beta=-\frac{1}{2} \arctan \left(\frac{2 \bar{\sigma}_{t}}{A}\right)
$$

From the values of $\bar{\sigma}_{n}$ and $\bar{\sigma}_{t}$ Equation( 4 ) and $\beta$ Equation (13), the stress $\bar{\sigma}_{I}$ can be determined by applying Equation( 6) $\left(\bar{\sigma}_{I}=\bar{\sigma}_{n}+\bar{\sigma}_{t} \tan \beta\right)$. But, for the stress $\bar{\sigma}_{I I}$, it is sensitive with small values of $\beta$. By introducing the values of the mean strains $\bar{\varepsilon}_{n n}$ and $\bar{\varepsilon}_{t t}$ Equation (5) and the value of $\sigma_{I}$ in Equation (10), we obtain

$$
\bar{\sigma}_{I I}=\frac{E}{1-v}\left(\bar{\varepsilon}_{n n}+\bar{\varepsilon}_{t t}\right)-\bar{\sigma}_{I}
$$

3.2.4. Failure criterion - calculation of effective stress intensity factor $K_{\theta \theta}$. The stress intensity factor $K_{\theta \theta}$ must be obtained for a certain direction $\theta_{0}$, orthogonal to the direction of the principal stress. The maximum circumferential tensile stress $\bar{\sigma}_{\theta \theta}\left(\theta_{0}\right)$ is then defined as (Figure10) follows:

$$
\left\{\begin{array}{l}
\bar{\sigma}_{\theta \theta}\left(\theta_{0}\right)=\bar{\sigma}_{I}, \text { if }|\beta| \leq \pi / 4 \\
\bar{\sigma}_{\theta \theta}\left(\theta_{0}\right)=\bar{\sigma}_{I I}, \text { if }|\beta|>\pi / 4
\end{array}\right.
$$

where $\bar{\sigma}_{I}$ and $\bar{\sigma}_{I I}$ are the mean principal stresses acting on two adjacent contacts (Figure 8(b)).

From the value of stress $\sigma_{\theta \theta}\left(r, \theta_{0}\right)$ close to a crack Equation (2), its mean value over a distance $0<r<d$ can be written of a function of $K_{\theta \theta}\left(\theta_{0}\right)$ as

$$
\bar{\sigma}_{\theta \theta}\left(\theta_{0}\right)=\frac{1}{d} \int_{0}^{d} \sigma_{\theta \theta}\left(r, \theta_{0}\right) d r=\frac{1}{d} \int_{0}^{d} \frac{K_{\theta \theta}\left(\theta_{0}\right)}{\sqrt{2 \pi r}} d r=\sqrt{\frac{2}{\pi d}} K_{\theta \theta}\left(\theta_{0}\right) .
$$

Using the value of $\bar{\sigma}_{\theta \theta}\left(\theta_{0}\right)$ obtained by the study of the forces on two contacts Equation (15), the value of the effective stress intensity factor $K_{\theta \theta}\left(\theta_{0}\right)$ is simply calculated (depending on the result of Equation( 16) as

$$
K_{\theta \theta}\left(\theta_{0}\right)=\bar{\sigma}_{\theta \theta}\left(\theta_{0}\right) \sqrt{\frac{\pi d}{2}}
$$

In practice, we calculate the stress intensity factor $K_{\theta \theta}\left(\theta_{0}\right)$ for each pair of neighbor contacts. The analysis of all contact pairs eliminates any difficulty of finding crack tips. When $K_{\theta \theta}\left(\theta_{0}\right)$ reaches the value of the material toughness $K_{I C}$, the cohesion forces of the most tensioned contact of the pair are set to zero, giving rise to the propagation of the crack at the proximity of this contact.

\subsection{Isotropic behavior}

The structure used in our discretization presents an angular period of $\pi / 3$ radians (Figure 11). However, this geometrical anisotropy describes coherent isotropic elasticity [22]. Our approach in fracture mechanics is based entirely on elasticity results. We may expect the same coherence on the crack analysis, with eventually some difference with the convergence. In a rough discretization, cracks may be described slightly differently considering the direction of the discrete structure. These differences tend to disappear with the refinement of the model. 

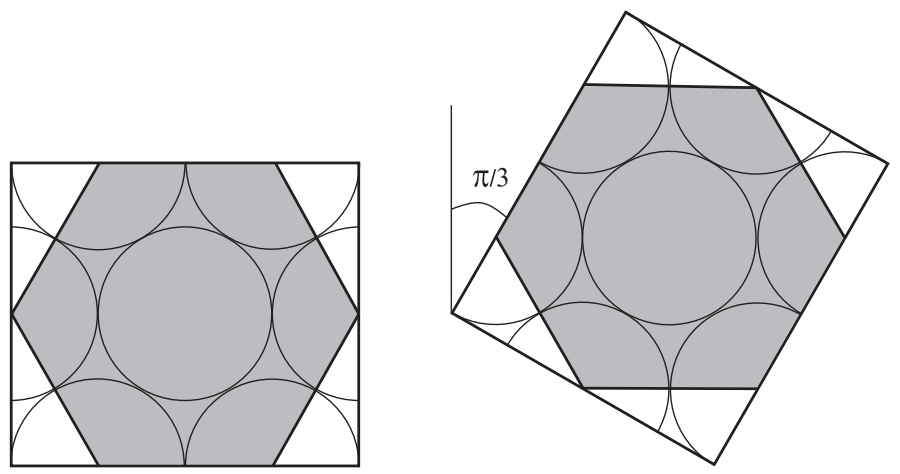

Figure 11. $\pi / 3$ geometrical periodicity.

\section{COMPARISON WITH CLASSICAL RESULTS}

In this section, we compare our numerical results to continuous solutions of fracture mechanics of brittle materials. Three situations are analyzed: pure tensile fracture, mixed mode beam crack, and biaxial fracture. The load intensity in all examples is driven by imposed displacements, which allow us to keep the crack propagation in quasi-static regime.

We suppose, for simplicity, that the particle diameter $d$ also corresponds to the thickness of the simulated plane stress geometries without loss of generality. The units of length and mass are respectively $d$ and the particle mass $m$. This implies (for a given material toughness $K_{I C}$ ) $T=\sqrt{m /\left(K_{I C} \sqrt{d}\right)}$ as time unit and $K_{I C} / \sqrt{d}$ as stress unit. Small strain is modeled taking $k_{n}=10^{4} K_{I C} / \sqrt{d}$ as normal stiffness. The ratio between tangential and normal stiffness $k_{t} / k_{n}=$ 0.5 (directly related to Poisson ratio) has no fundamental effect in plane stress results. We adopt a small value of viscous damping $c_{n}=0.65 \sqrt{m K_{I C} \sqrt{d}}$.

\subsection{Tension panel}

We present the results for two pre-cracked samples: middle-crack tension panel (Figure 12(a)) and double-edge notch tension panel (Figure 12(b)), which are classical examples of tensile (mode I) crack. A vertical displacement induces a mean tensile stress $\Sigma$ to the sample. We measure the maximum stress value $\Sigma^{\max }$ supported by the structure before complete fracture of the panel. The panels have a rectangular shape with height equal to three times width $L$ to avoid eventual boundary effects.
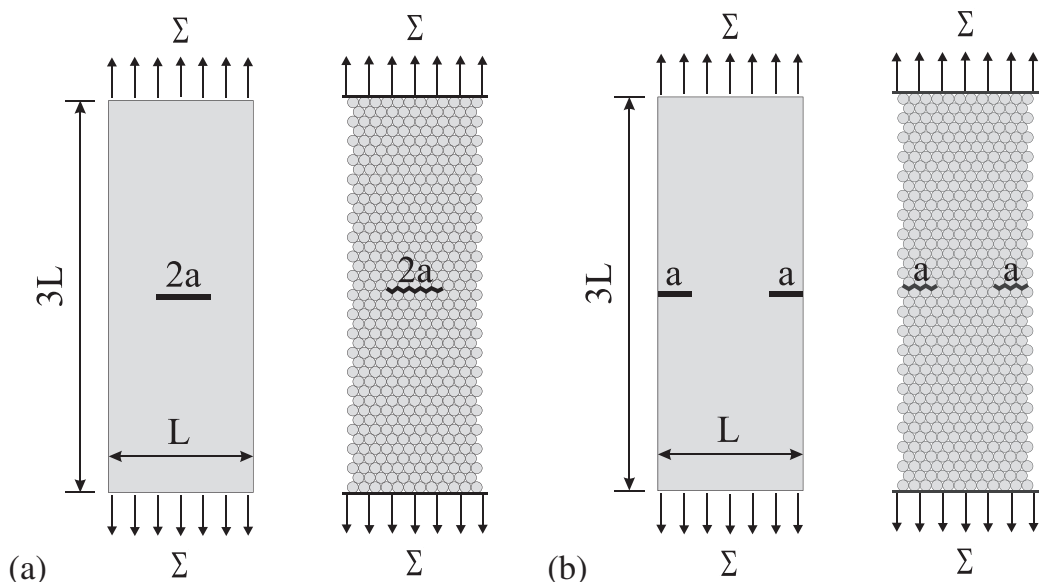

(b)

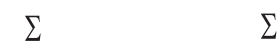

Figure 12. Tensile test: (a) middle-crack panel and (b) double-edge notch panel. The discrete samples correspond to $L / d=11$. 
Our numerical results of $\Sigma^{\max }$ are compared with the following expressions [29]:

- middle-crack tension panel

$$
\Sigma^{\max }=\frac{K_{I C}}{\sqrt{\pi a}} \sqrt{\cos \frac{\pi a}{L}}\left[1-0.1(a / L)^{2}-0.96(a / L)^{4}\right]^{-1},
$$

- double-edge notch tension panel

$$
\Sigma^{\max }=\frac{K_{I C}}{\sqrt{\pi a}} \sqrt{1-\frac{2 a}{L}}\left[1.122-1.122(a / L)-0.82(a / L)^{2}+3.768(a / L)^{3}-3.04(a / L)^{4}\right]^{-1} .
$$

In both cases, four different crack lengths $a / L$ were tested: $3 / 22,4 / 22,5 / 22$, and $6 / 22$. The maximum stress $\Sigma^{\max }$ supported by the panels before fracture propagation decreases when crack length grows as shown in Figure 13. The systematic variation of the ratio $L / d$ allows the analysis of the discrete approach convergence. Higher values of $L / d$ are associated with more precise descriptions of the crack zone inducing better results whatever the crack type. We observe an error of $10-15 \%$ for $L / d=11$, which decreases to less than $5 \%$ for $L / d=22$ and keep decreasing for subsequent increase of $L / d$.

A consistent isotropic description is supported by the results obtained with a discretization presenting an angular offset of $\pi / 6$ rad for $L / d=88$ (as discussed in Section 3.3, this offset corresponds to half of the angular period of $\pi / 3 \mathrm{rad}$ of the discrete structure). This absence of the effect of the orientation of the discrete structure for two extreme cases ( $\varphi=0$ and $\varphi=\pi / 6$ radians) is a strong indicator of isotropic behavior described by the failure criterion, and this is confirmed with the study of crack propagation in mixed mode in the following sections.
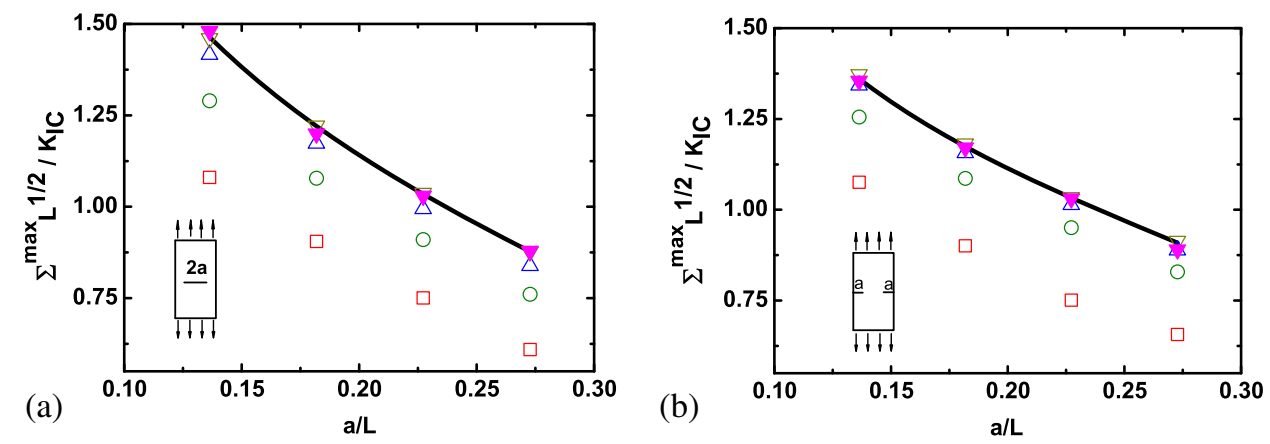

Figure 13. Normalized maximum stress $\Sigma^{\max } \sqrt{L} / K_{I C}$ as a function of the crack length $a / L$ for the (a) middle-crack panel and (b) double-edge notch panels with different discretizations: ( $\square$ ) $L / d=11$, (o) $L / d=22,(\triangle) L / d=44,(\nabla) L / d=88$, and $(\nabla) L / d=88$ with an angular offset of the particle structure of $\pi / 6$ radians. The continuous lines indicate the reference expressions for $\Sigma^{\max }$ corresponding to Equations (18) and (19).

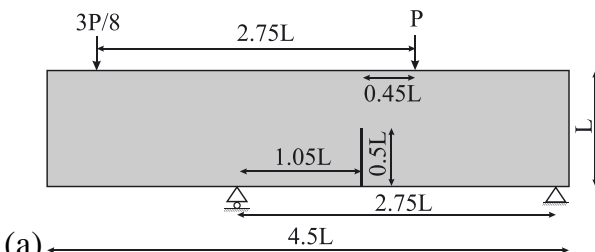

(a)

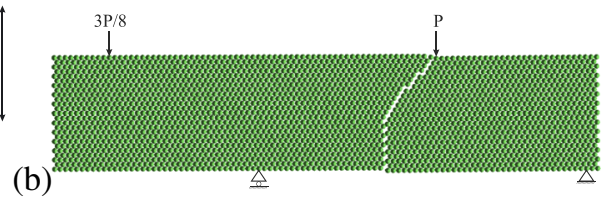

Figure 14. (a) Pre-cracked beam setup and (b) collapsed structure for $L / d=22$. 


\subsection{Pre-cracked beam}

The crack tip of the pre-cracked beam in Figure 14(a) is under mixed mode (I and II). The stress intensity factors can be written as $K_{I}=8.76 M \sqrt{\pi a} / L^{2}$ and $K_{I I}=1.39 \mathrm{~V} \sqrt{\pi a} / L$ [30], where $L$ is the beam height, $a=0.5 L$ is the crack length, $M$ and $V$ are the bending moment and the shear force, respectively. According to Figure 14(a), $M=0.1875 L P$ and $V=0.625 P$, both are proportional to the force per unit of length $P$, applied over the beam thickness by imposed displacements. Following the maximum circumferential tensile stress, the limit $K_{\theta \theta}\left(\theta_{0}\right)=K_{I C}$ imposes a maximum value for $P, P_{\max } \approx 0.37 K_{I C} \sqrt{L}$ before the collapse of the structure, shown in Figure 14(b) for a rough discretization. The crack propagation angle associated $\theta_{0} \approx 41.4^{\circ}$ Equation (A.3) corresponds nearly to the path from the crack tip until the loading force $P$.

We have tested three different discretizations $L / d: 22,44$, and 88 . We obtain a limit load of $90 \% P_{\max }$ with the first (and roughest) one. The two other leads respectively to $95 \%$ and $98 \%$ of $P_{\max }$, indicating a good convergence to the theoretical value.

\subsection{Biaxial fracture}

As previously seen for the pre-cracked beam, shear stress may induce crack deflection. Let us consider a square plate (side length $L$ ) under biaxial stresses (lateral compression $\Sigma_{x}$ and vertical tension $\Sigma_{y}$-controlled by the displacements of non-frictional moving walls) with an initial inclined crack of length $a$ (Figure 15(a)). Depending on the initial crack angle $\alpha$, a pure shear stress condition (in $\alpha$ direction) can be obtained if $\Sigma_{x} / \Sigma_{y}=\tan ^{2} \alpha$. Following the maximum circumferential tensile stress criterion (Section 3.1), for pure shear stress $\left(K_{I}=0\right)$, the angle which maximizes $\sigma_{\theta \theta}$ (Equation (A.3) in Appendix A) is $\theta_{0} \simeq 70.5^{\circ}$ (relative to $\alpha$ ). The crack deflection angle $\theta_{0}$ is only a prediction of the initial crack propagation. The general tendency of the crack is to become horizontal, orthogonal to stress tensile direction.

The results of the simulation of an inclined crack with $\alpha=60^{\circ}, a / L=0.41, L / d=176$ are shown in Figure 15(a) and (b). The crack propagation presents a coherent path tending slightly to a

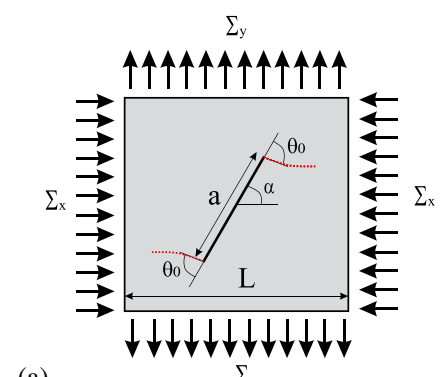

(a)

$\sum_{\mathrm{y}}$

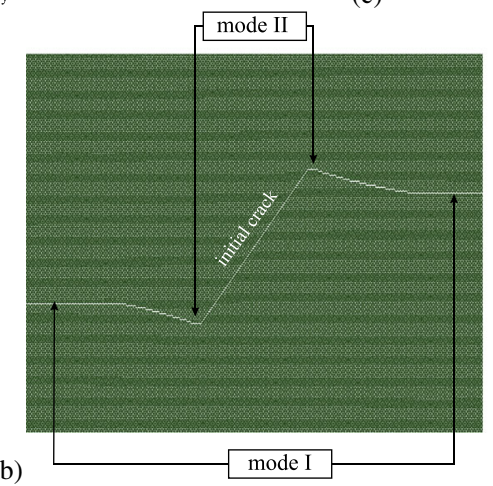

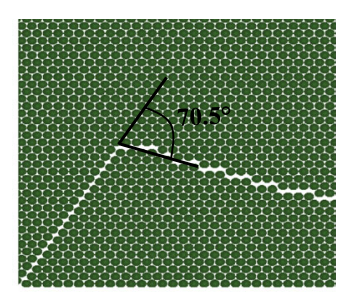

(c)

(b)

Figure 15. (a) Square plate with an inclined crack under biaxial loading. The dotted lines indicate the crack propagation path. (b) Simulated inclined crack under biaxial load. In detail, (c) the theoretical prediction of the crack branching. 
horizontal direction (Figure 15(a)). In detail, in Figure 15(b), the theoretical prediction of the initial crack deflection is fairly obtained.

\section{CONCLUSIONS}

We propose in this paper a DEM approach in fracture mechanics adapted to the analysis of isotropic brittle materials, which is entirely compatible with continuum classical theory. Based on the equivalence between discrete and continuum approaches in elasticity, we determine the tensors of stress and strain as a function of the forces and displacements of two adjacent contacts. It allows us to calculate the stress intensity factor $K_{\theta \theta}$ (in polar coordinates) and introduce the toughness of the material as a model parameter without any previous calibration, which represents an important feature for DEM models.

The consistency of the formulation is verified by numerical simulations; the DEM approach is first applied to two tensile cracks (mode I) and a pre-cracked beam (mixed mode). In these cases, the maximum load before the collapse of the structures is compared with theoretical solutions, wherein a fast monotone convergence as function of the discretization (particle size) is observed.

A usual drawback of discrete approaches is the anisotropy due to the particle structure adopted on the models, specially for regular patterns [25]. This undesirable effect on the study of isotropic materials is avoided in our formulation as shown by the results in mode I; two different orientations of the structure produce similar results. The biaxial test of the sample with an initial inclined crack under pure shear stress (mode II) also indicates a reduced effect of the particle structure on the crack branching, which follows the theoretical prediction. An isotropic behavior (despite the anisotropic structure of the discrete model) is essential to extend the scope to crack initiation and associate the strength of the material as a model parameter, as shown by Le [31].

\section{APPENDIX: $\theta_{0}$ AS FUNCTION OF $K_{I}$ AND $K_{I I}$ FOR THE MAXIMUM CIRCUMFERENTIAL STRESS CRITERIUM}

The angle $\theta_{0}$ can be derived for known stress intensity factors $K_{I}$ and $K_{I I}$. Based on Equation (2) and considering the maximum circumferential stress criterion $\left(K_{r \theta}\left(\theta_{0}\right)=0\right)$, we can write

$$
\frac{K_{I I}}{K_{I}}=\frac{-\sin \frac{\theta_{0}}{2} \cos ^{2} \frac{\theta_{0}}{2}}{\frac{1}{4} \cos \frac{\theta_{0}}{2}+\frac{3}{4} \cos \frac{3 \theta_{0}}{2}}=\frac{\sin \theta_{0}}{1-3 \cos \theta_{0}} .
$$

Equation (A.1) can be directly solved for $\theta_{0}$ by numerical methods or developed analytically as follows.

If we expand Equation (A.1) and divide the whole expression by $\sqrt{K_{I}^{2}+\left(3 K_{I I}\right)^{2}}$, we have

$$
\sin \theta_{0} \cos \phi+\cos \theta_{0} \sin \phi=\sin \left(\theta_{0}+\phi\right)=\frac{K_{I I}}{\sqrt{K_{I}^{2}+\left(3 K_{I I}\right)^{2}}},
$$

where $\cos \phi=K_{I} / \sqrt{K_{I}^{2}+\left(3 K_{I I}\right)^{2}}$ and $\sin \phi=3 K_{I I} / \sqrt{K_{I}^{2}+\left(3 K_{I I}\right)^{2}}$.

Finally, the exact value of $\theta_{0}$ can be obtained by the inversion of Equation (A.2) as

$$
\theta_{0}=\arcsin \left(\frac{K_{I I}}{\sqrt{K_{I}^{2}+\left(3 K_{I I}\right)^{2}}}\right)-\phi,
$$

where $\phi=\arccos \left(K_{I} / \sqrt{K_{I}^{2}+\left(3 K_{I I}\right)^{2}}\right)=\arcsin \left(3 K_{I I} / \sqrt{K_{I}^{2}+\left(3 K_{I I}\right)^{2}}\right)$. 


\section{REFERENCES}

1. Barenblatt GI, Salganik RL, Cherepanov GP. On the nonsteady motion of cracks. PMM Applied Mathematics and Mechanics 1962; 26:469-477.

2. Dougadale D. Yielding of steel sheets containing slits. Journal of the Mechanics and Physics of Solids 1960; 8(2):100-104.

3. Henshell RD, Shaw KG. Crack tip finite elements are unnecessary. International Journal for Numerical Methods in Engineering 1975; 9(3):495-507.

4. Nicolas Moës, Dolbow J, Belytschko T. A finite element method for crack growth without remeshing. International Journal for Numerical Methods in Engineering 1999; 46(1):131-150.

5. Moës N, Gravouil A, Belytschko T. Non-planar 3D crack growth by the extended finite element and level sets, part i : mechanical model. International Journal for Numerical Methods in Engineering 2002; 53:2549-2568.

6. Abdelaziz Y, Hamouine A. A survey of the extended finite element. Computers and Structures 2008; 86 (11-12):1141-1151

7. Cundall PA, ODL Strack. A discrete numerical model for granular assemblies. Gotech 1979; 29:47-65.

8. Morgan JK, Boettcher MS. Numerical simulations of granular shear zones using the distinct element method-1, Shear zone kinematics and the micromechanics of localization. Journal of Geophysical Research 1999; 104(B2): 2703-2719.

9. Cleary P. Modeling comminution devices using DEM. International Journal for Numerical Methods in Geomechanics 2001; 25:83-105.

10. Jensen RP, Plesha ME, Edil TE, Bosscher PJ, Kahla NB. DEM simulation of particle damage in granular mediastructure interfaces. The International Journal of Geomechanics 2001; 1(1):21-39.

11. Viggiani F, Calvetti G, Tamagnini C. A numerical investigation of the incremental non-linearity of granular soils. Italian Geotechnical Journal, Special Issue on Mechanics and Physics of Granular Materials 2003; 37(3):11-29.

12. Sibille L, Nicot F, Donzé FV, Darve F. Material instability in granular assemblies from fundamentally different models. International Journal For Numerical and Analytical Methods in Geomechanics 2007; 31:457-481.

13. Koval G, Roux JN, Corfdir A, Chevoir F. Annular shear of cohesionless granular materials: from the inertial to quasistatic regime. Physical Review E 2009; 79(2):021306.

14. Meguro K, Hakuno M. Fracture analysis of concrete structures by the modified distinct element method. Structural Engineering / Earthquake Engineering 1989; 6(2).

15. Takada S, Hassani N. Earthquake Resistant Engineering Structures, vol. 23, Analysis of compression failure of reinforced concrete by the modified distinct element method. Computational Mechanics Publications: Southampton, UK, 1996.

16. Matsuda Y, Iwase Y. Numerical simulation of rock fracture using three-dimensional extended discrete element method. Earth Planets Space 2002; 54:367-378.

17. Hentz S, Daudeville L, Donzé FV. Identification and validation of a discrete element model for concrete. Journal of Engineering Mechanics ASCE 2004; 130(6):709-719.

18. Potyondi DO, Cundall PA. A bonded-particle model for rock. International Journal of Rock Mechanics and Mining Sciences 2004; 41(8):1329-1364.

19. Miehe C, Dettmar J, Zäh D. Homogenization and two-scale simulations of granular materials for different microstructural constraints. International Journal for Numerical Methods in Engineering 2010; 83:1206-1236.

20. Wang YN, Tono F. Calibration of a discrete element model for intact rock up to its peak strength. International Journal for Numerical and Analytical Methods in Geomechanics 2010; 34:447-469.

21. Grima AP, Wypych PW. Development and validation of calibration methods for discrete element modelling. Granular Matter 2011; 13:127-132.

22. Tavarez FA, Plesha ME. Discrete element method for modelling solid and particulate materials. International Journal for Numerical Methods in Engineering 2007; 70:379-404.

23. Wang Y, Mora P. Macroscopic elastic properties of regular lattices. Journal of the Mechanics and Physics of Solids 2008; 56:3459-3474.

24. Allen MP, Tildesley DJ. Computer Simulation of Liquids. Oxford University Press: Oxford, 1987.

25. Le BD, Koval G, Chazallon C. Discrete element approach in brittle fracture mechanics. Engineering Computations 2013; 30(2):263-276.

26. Irwin G. Analysis of stresses and strains near the end of a crack traversing a plate. Journal of Applied Mechanics 1957; 24:361-364.

27. Labbens R. Introduction à la mécanique de la rupture. Éditions Pluralis: Paris, 1980.

28. Erdogan F, Sih GC. Crack extension in plates under plane loading and transverse shear. Journal of Basic Engineering $1963 ; 85(4): 519-527$.

29. Tada H, Paris PC, Irwin GR. Stress analysis of cracks handbook. Paris productions: Saint Louis, MO, 1985.

30. Balloch RA, Brown MW. The effect of pre-cracking history on branch crack threshold under mixed mode I/II loading. In Fatigue under biaxial and multiaxial loading, Kussmaul K, McDiarmid D, Socie D (eds). Mech. Pub.: London, 1991; 179-197.

31. Le BD. Modélisation discréte en mécanique de la rupture des matériaux fragiles. PhD thesis, INSA de Strasbourg, France, 2013. 